\title{
New solutions in precision lens mounting
}

\author{
Frédéric Lamontagne ${ }^{1}\left[\mathbb{D} \cdot\right.$ Nichola Desnoyers $^{1}$
}

Received: 30 January 2019 / Accepted: 3 June 2019 / Published online: 10 June 2019

(c) The Author(s) 2019

\begin{abstract}
Several methods have been developed through the years to mount lenses with the ultimate goal of minimizing their positioning errors with respect to the nominal optical layout. This is a non-trivial task, since it requires either very well controlled manufacturing tolerances or alignment of the optical components into their mounts. This paper reviews the classical lens mounting methods and introduces new solutions to improve the centering accuracy. First, an improved drop-in method called auto-centering is described. This method is based on the use of geometrical relationship between the lens diameter, the lens radius of curvature, and the thread angle of the retaining ring to provide centring error typically less than $0.5 \mathrm{arcmin}$. In addition, an innovative method that relies on geometric principles to auto-center optomechanical parts to each other is described. The method allows to auto-center an optical group in a main barrel, to perform an axial adjustment of an optical group inside a main barrel, and to perform stacking of multiple barrels within $5 \mu \mathrm{m}$ of centering error. Finally, to improve the centering accuracy of common method used to center injection molded plastic lenses, a new concept using toroidal interfaces has been developed. This method allows a reduction by at least a factor of two the lens centering error compared to methods based on radial clearance fit for the same manufacturing tolerances.
\end{abstract}

Keywords Lens mounting $\cdot$ Auto-centering $\cdot$ Molded lens $\cdot$ Alignment $\cdot$ Centering $\cdot$ Optical mount

\section{Introduction}

In most common lens mounting methods, the lens is simply dropped into a barrel and secured in place with a retainer or an adhesive [1]. The positioning error of the lens is controlled by manufacturing tolerances without any alignment. With this mounting method called drop-in assembly, several parameters influence the lens positioning accuracy. First, radial clearance between the lens and the barrel needs to be considered. The centering error of the optical surfaces of the lens with respect to the lens datum axis also affects the lens centering relative to the barrel axis. If the lens is mounted on a non-optical surface, the geometric error between the mounting surface and the optical axis of the lens will also affect the centering. Another contributor to the lens centering is the manufacturing errors of the barrel. The perpendicularity and the concentricity of the lens seat, as well as

Frédéric Lamontagne

frederic.lamontagne@ino.ca

1 INO, 2740 Einstein Street, Quebec, QC, Canada the concentricity of the barrel inner diameter relative to the barrel reference axis are among the main contributors. Moreover, when the lens is mounted on a spherical surface instead of a planar surface, the lens rolls rather than translates, causing a centering error of the lens plus a tilt. Figure 1 shows an example of a lens mounted in a barrel including all the manufacturing error listed above.

In the case of aspheric lens, the manufacturing error of the aspheric surface with respect to the other optical surface must also be considered. In fact, aspheric lens present additional challenges, since the centering and tilt manufacturing error between the two optical surfaces cannot be fully compensated either by the mounting or by alignment [3].

When the centering requirements are at the tolerancemanufacturing limit for the drop-in method, other strategies to align the lens need to be considered. Most of the time, active alignment techniques such as aligning a lens directly in a barrel, sub-cell assembly, or alignment in 5 degrees of freedom with sophisticated equipment are some of the solutions envisioned. All these different implementations of the active alignment provide high-centring accuracy, but also 


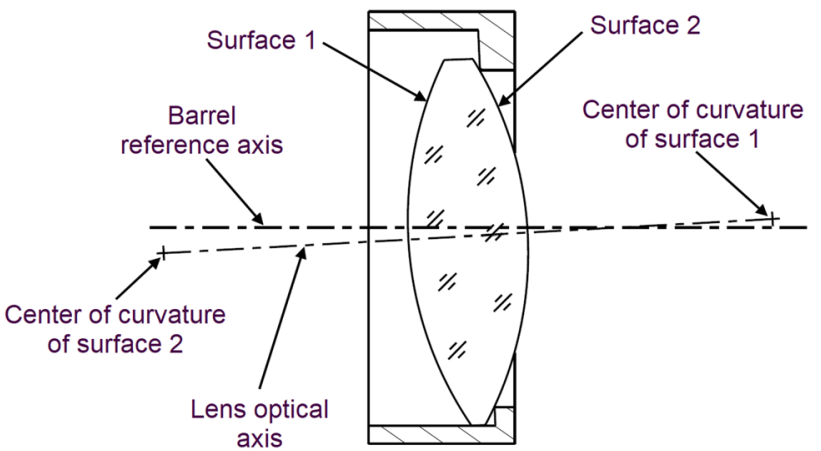

Fig. 1 Lens positioning error considering all main contributors [2]

require expensive equipment and more assembly steps to perform the alignment, resulting in a cost increase.

\section{Lens auto-centering}

The drop-in method as described previously relies on the lens outer cylindrical diameter to center the optical axis with respect to the barrel reference axis. This method refers to rim-contact mounting. Surface-contact mounting is a second type of drop-in method, where the optical component is constrained in the barrel using the optical surfaces instead of the lens rim. Mounting the lens directly on optical surfaces has the advantage of not requiring tight diameter tolerances and accurate lens edging, i.e., centering of the lens optical surface with respect to the lens outer diameter. To provide a good centering accuracy with surface-contact mounting, two conditions must be fulfilled. First, the clamping angle at the contact between the lens optical surfaces and the mounting seats must be large enough to overcome the friction forces when an axial force is applied on the optical surfaces. The minimum clamping angle that allows a lens to be self-centered in a lens barrel using a threaded ring as retainer has been determined experimentally [4]. Different optical coatings and anodic processes have been tested to determine a typical worst case-clamping angle for common coatings. These measurements have shown that a clamping angle of $14^{\circ}$ was sufficient to self-center lenses for all the optical coating and anodic process combinations tested. The centerability criterion to self-center a lens in a barrel using surface-contact mounting can be expressed as

$\sin ^{-1}\left(\frac{Y_{\mathrm{c} 1}}{R_{1}}\right)+\sin ^{-1}\left(\frac{Y_{\mathrm{c} 2}}{R_{2}}\right) \geq 14^{\circ}$,

where $Y_{\mathrm{cl}}(\mathrm{mm})$ is the half-diameter of contact of the first surface $\mathrm{S} 1$ of the optical element with the barrel seat, $Y_{\mathrm{c} 2}$ $(\mathrm{mm})$ is the half-diameter of contact of the second surface $\mathrm{S} 2$ of the optical element with the retaining ring, $R_{1}(\mathrm{~mm})$ is

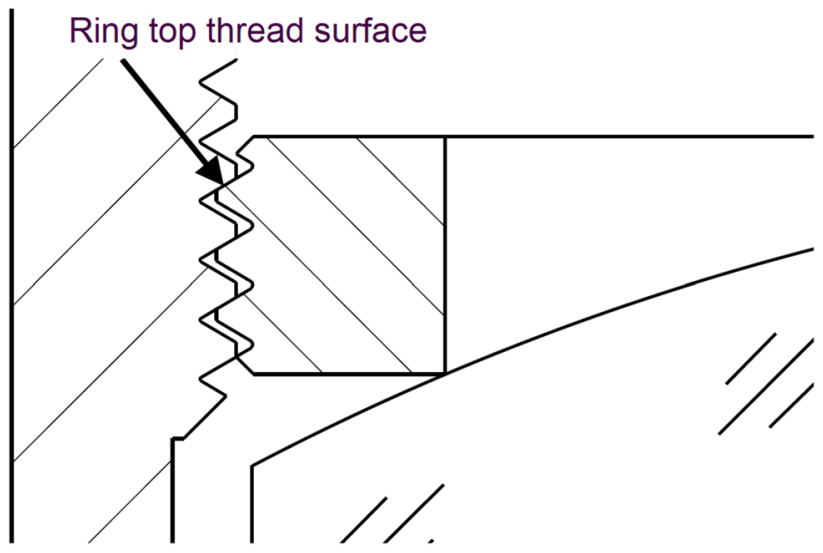

Fig. 2 Threaded ring constrained by the top thread surface

the radius of curvature of the first surface of the optical element, and $R_{2}(\mathrm{~mm})$ is the radius of curvature of the second surface of the optical element. The radius of curvature has a positive value for convex surfaces and a negative value for concave surfaces for the clamping angle equation.

The second condition to get good centering accuracy with surface-contact mounting is that both lens mounting seats must be concentric and perpendicular to the barrel reference axis. This condition can be easily satisfied for the barrel-mounting seat, since very small concentricity and perpendicularity manufacturing errors are easy to achieve for a single setup machining process on computer numerical control (CNC) lathe. Measurements performed on lens having diameters ranging from 10 to $75 \mathrm{~mm}$ and having different radii of curvature have shown that centering errors are generally below 0.25 arcmin when the barrel seat is manufactured in the same machining setup as the barrel mechanical axis. Unfortunately, this is not as simple for the other lensmounting seat, which is often provided by a retainer. One of the most common retainers used to secure a lens in a barrel is the threaded ring. Because of the thread assembly clearance, the ring provides a mounting seat for the lens that is decentered and tilted. As a result, the standard threaded rings provide in most cases poor centering accuracy for surfacecontact lens mounting. Experimental measurements have shown that centering errors of lens surface in contact with threaded ring vary up to 10 arcmin for typical lens assembly. To have a good understanding on how the thread angle affects the ring tilt error, we need to be aware that the ring thread is constrained by its top thread surface within the barrel thread. In fact, when a ring is rotated to secure a lens in a barrel, an axial force constrained the lens on the barrel seat and a reaction force pushes the ring on the opposite side so that the ring is constrained by its top thread surface, as shown in Fig. 2.

Since the ring is constrained on the top thread surface, the ring rolls according to the thread angle when 


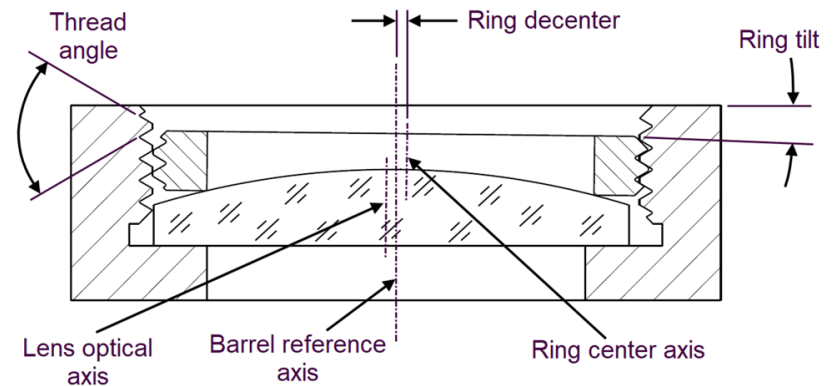

Fig. 3 Relationship between the ring decenter and ring tilt

decentered, as illustrated in Fig. 3. This rolling movement of the ring means that the ring tilt and its lateral decentering are linked to each other in a geometrical manner. As a result, the lens seat of the ring is decentered and tilted because of the threads assembly clearance. As discussed previously, the lens is positioned as per the ring lens seat if the criterion of centerability is met. It can be seen in Fig. 3 how the ring seat positioning error caused by the thread assembly clearance affect the lens centering.

To overcome this issue, an improved drop-in method called the auto-centering has been developed recently [4]. This method is based on the use of the geometrical relationship between lens diameter, lens radius of curvature, and the thread angle of the retaining ring. The barrel thread angle and the spatial profile of the peripheral region of the lens surface are selected to create auto-centering conditions, whereby any decentering of the retaining ring and a corresponding tilt of the retaining ring have counterbalancing effects on the centering of the optical element. Thus, the lens is maintained aligned independently of the ring positioning error. The thread angle to meet the autocentering condition for a given lens geometry is defined by the following equation:

$\varphi_{\text {threads }}=2 \tan ^{-1}\left(\frac{d_{\text {ring }}}{2 \sqrt{R^{2}-Y^{2}}+2 h+T}\right)$,

where $d_{\text {ring }}$ is the major diameter of the retaining ring, $R$ is the radius of curvature of the lens surface in contact with the retaining ring, $Y$ is the half-diameter of the retaining ring's clear aperture, $h$ is the distance between the lens point of contact with the ring and the first thread, and $T$ is a parameter associated with the ring thickness [5]. Figure 4 shows a lens barrel assembly, where the auto-centering condition is met. It can be seen that the retaining ring is decentered and tilted within the thread assembly clearance. However, even if the ring has a positioning error, the lens is still centered on the barrel reference axis, since the auto-centering condition is met.

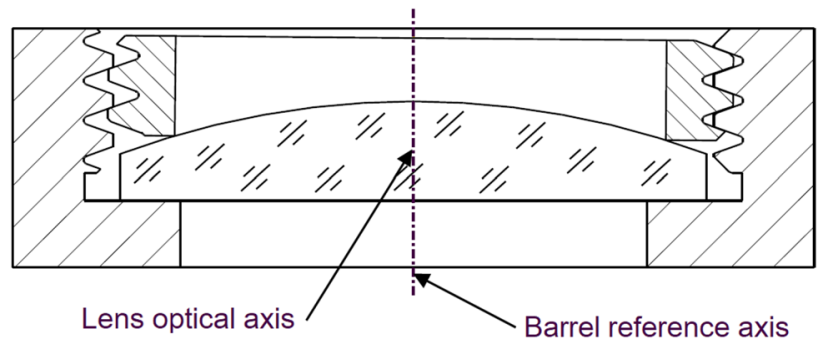

Fig. 4 Auto-centered lens not affected by the ring positioning error

Experimental measurements have demonstrated that the auto-centering method results in centering errors typically lower than 0.5 arcmin for the lens surface in contact with the ring. This new lens mounting method has the advantage of providing very accurate centering while relaxing the manufacturing tolerances on lens wedge, lens diameter, and barrel bore diameter as required for the precision drop-in method. Using different thread shape, the auto-centering method allows centering of convex, planar, concave, and aspheric lenses.

\section{Aspheric lens auto-centering}

In the case of aspheric lens, the same equation as for spherical lenses can be applied to determine the auto-centering thread angle using the local radius of curvature at the ring interface diameter [6]. Figure 5 shows an example of a lens having two aspheric surfaces mounted with the auto-centering method. Exaggerated manufacturing errors on decenter and tilt of the aspheric surfaces are shown in the figure. The barrel seat constrains the aspheric surface 2 , so that the center of curvature of the local radius at the barrel seat interface diameter (point 5) is centered on the barrel reference axis. The threaded ring on the left side of the figure constraints and auto-centers the aspheric surface 1, so that the center of curvature of the local radius at the threaded ring interface diameter (point 2) is also centered on the barrel reference axis. The centering errors of the paraxial spheres of both lens surfaces result from the lens manufacturing errors on the aspheric surfaces decenter and tilt with respect to each other.

The difference between active alignment and auto-centering of aspheric lens is that the centering is done at the center of curvature of the local radius interfacing with the ring (point 2) for the auto-centering rather than at the paraxial sphere center of curvature (point 3 ) for the active alignment. Figure 6 shows an example of a lens having a spherical surface mounted on the barrel seat and an aspheric surface aligned with a centering machine, so that the paraxial sphere (point 3 ) is centered on the barrel reference axis. 
Fig. 5 Auto-centered biaspheric lens
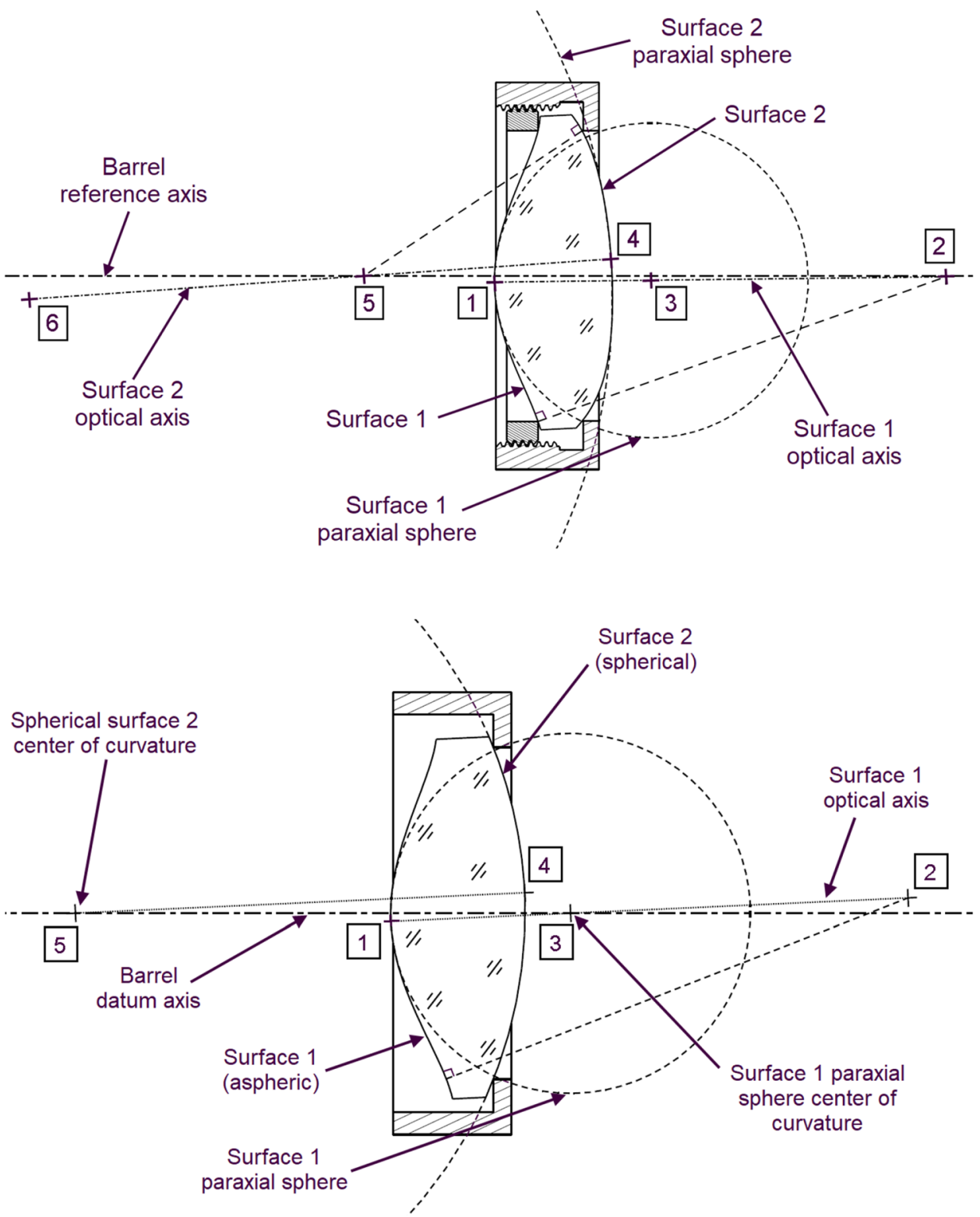

Fig. 6 Aspheric-spherical lens active alignment
For both aspheric centering methods, it is unfortunately impossible to provide a perfect centering because of the decenter and tilt manufacturing errors between the aspheric surface and the optical surface in contact with the barrel seat.

Centering measurements for different aspheric lenses mounted using the auto-centering have shown that this new lens mounting method for aspheres provides a simple and an accurate mounting method that bridges the advantages of the standard drop-in and the active alignment.

\section{Precision optomechanical assembly}

In addition to lens centering, the approach that uses threads as mechanical reference also allows to auto-center an optical group in a main barrel, performs an axial adjustment of an optical group inside a main barrel, and performs stacking of multiple barrels. Experimental measurements have shown that this mounting method using threads to align optical sub-assembly results in centering errors usually smaller than $5 \mu \mathrm{m}$. All these implementations are shown in Fig. 7 [7]. 
Fig. 7 Lens barrel assembly using thread as mechanical reference

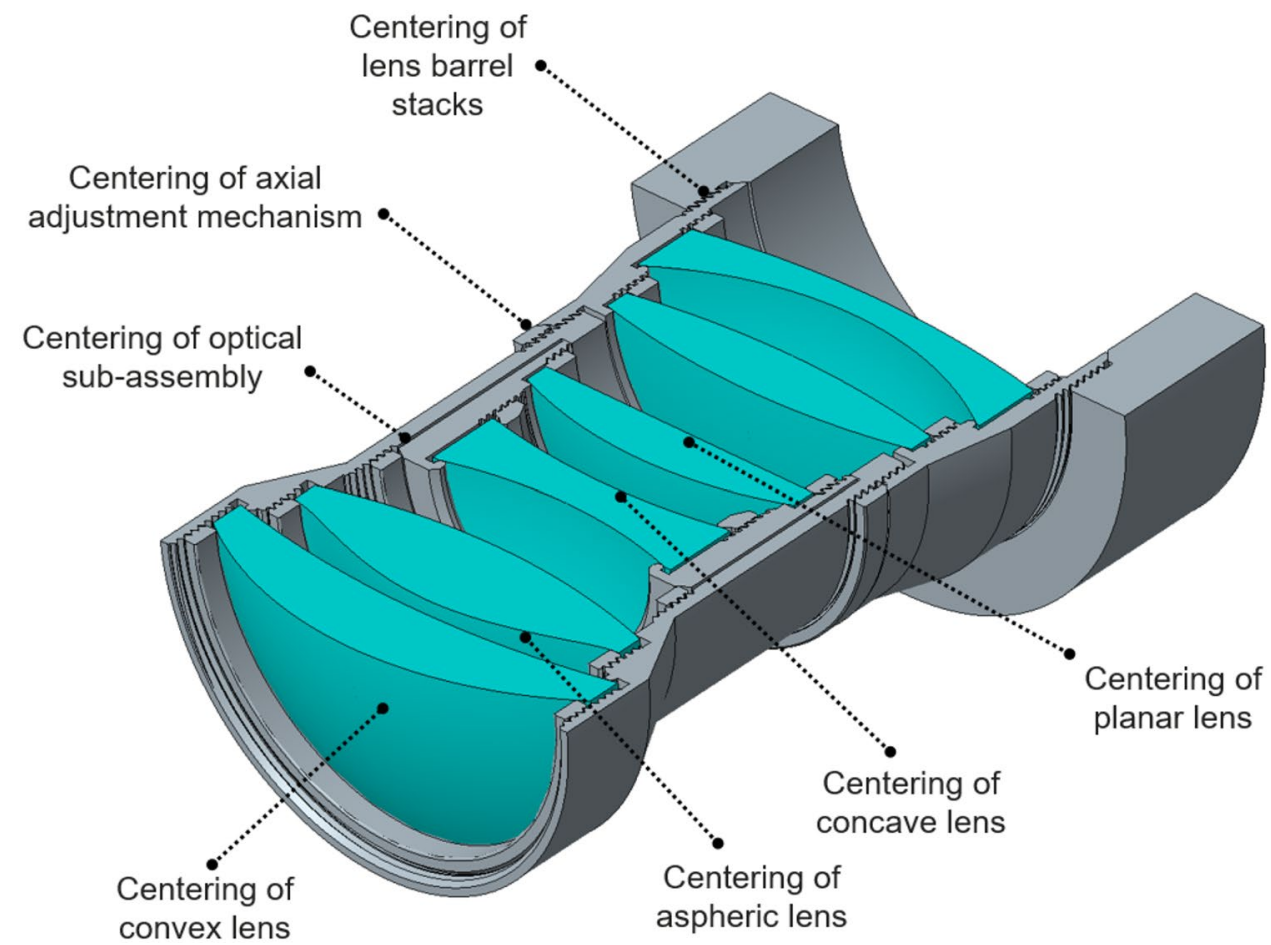

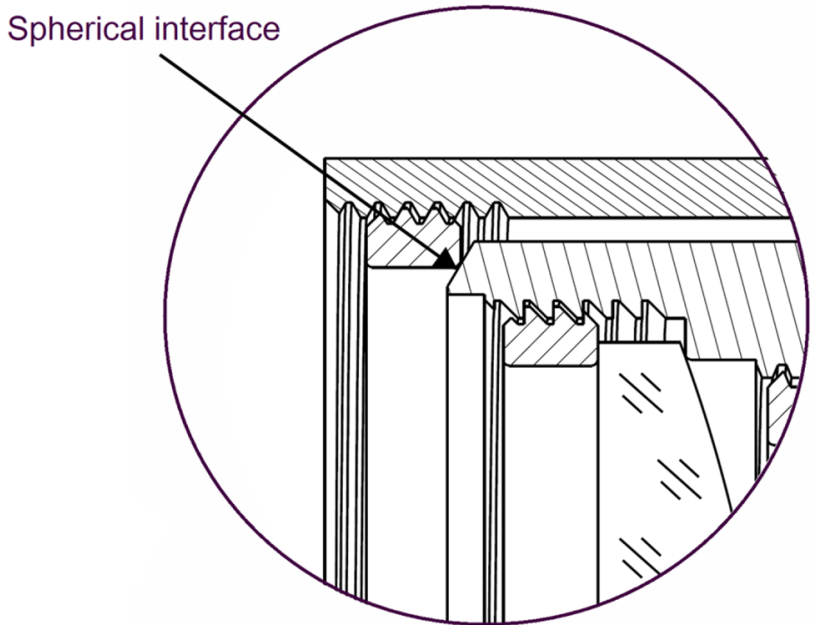

Fig. 9 Auto-centering of optical sub-assembly zoomed view

the spherical interface and the ring thread used to meet the auto-centering condition.

\subsection{Translatable optical group}

An axial adjustment of an optical group is often used as compensator in optical systems. Adding this degree of freedom makes it challenging to keep the centering of the translatable assembly over the moving range. The use of thread also allows to combine the centering of an optical 


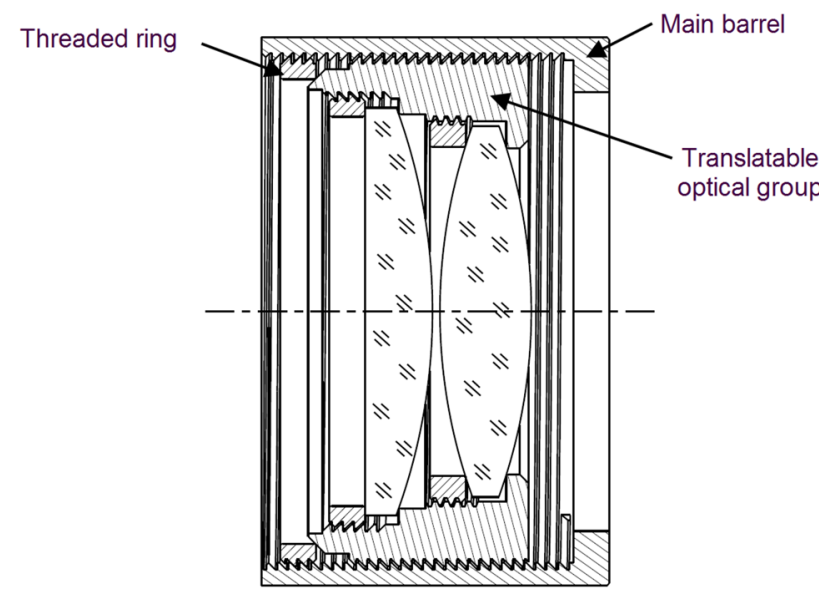

Fig. 10 Auto-centering of translatable optical group

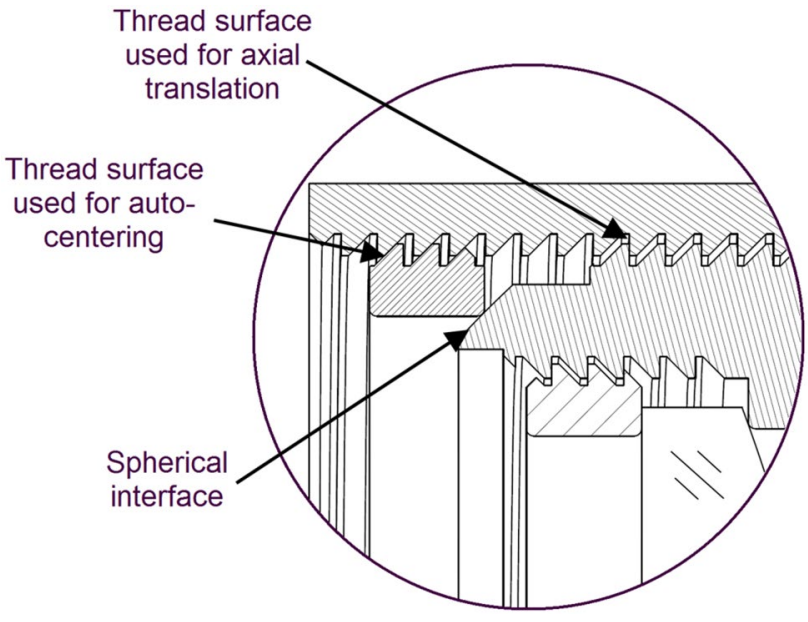

Fig. 11 Auto-centering of translatable optical group zoomed view

sub-assembly with an axial adjustment within a main barrel. A buttress thread is used for the axial displacement. This kind of thread has the particularity to have a face that is perpendicular to the barrel reference axis. Since this face is used for the axial translation, any centering error of the moving barrel does not impart tilt on the optical sub-assembly, as for the optical sub-assembly, as shown in Fig. 8, where the seat is fixed and perpendicular to the barrel reference axis. The centering of the translatable group is then provided once the axial adjustment is performed by the use of a threaded ring and a spherical interface that meets the auto-centering condition. When the ring is tightened on the moving barrel to lock the axial position, the sub-assembly translates on the thread surface perpendicular to the barrel reference axis to be centered on the main barrel reference axis. Figures 10 and 11 show the details of this implementation of the autocentering on a translatable optical group.

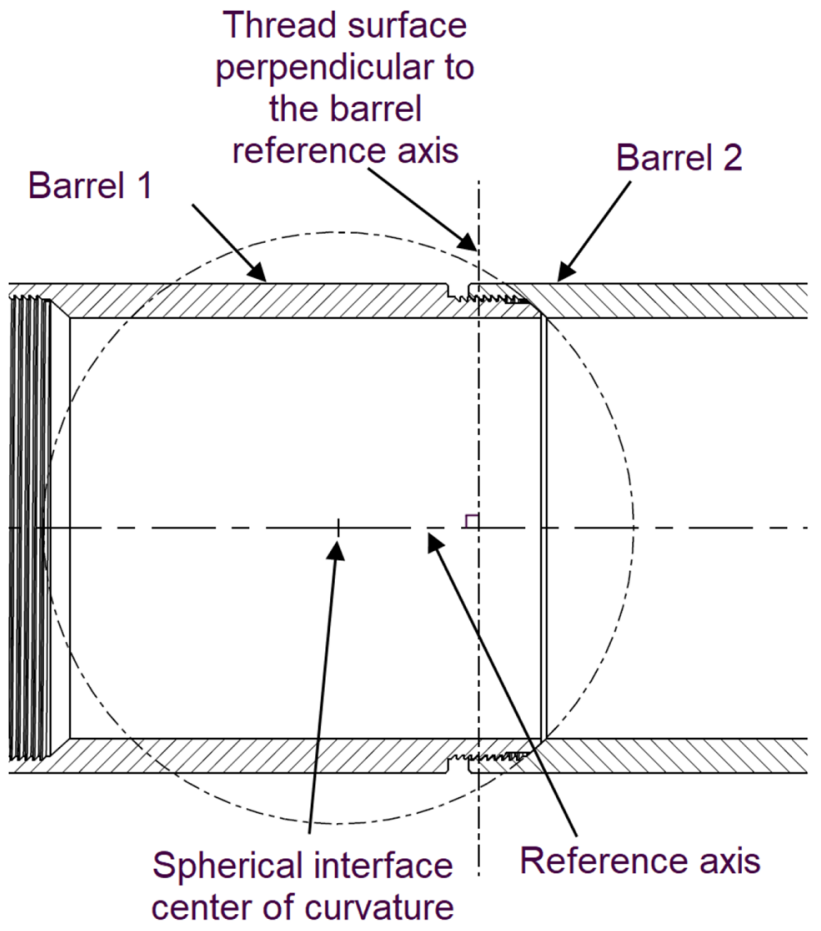

Fig. 12 Barrel stack auto-centering

\subsection{Barrel stack auto-centering}

There is often a need in optomechanical design to mount lens barrels to each other. This is the case when an iris or a reticle is mounted between two optical groups in a lens assembly. Once again, threaded interface offers a high accuracy mounting method without the use of tight dimensional tolerance on pilot diameters as commonly used in classical optical assemblies. In this implementation, the two barrels interface with each other through a spherical interface. This provides a very accurate centering of the center of curvature of the first barrel spherical interface with respect to the second barrel reference axis. Then, the remaining rotation degrees of freedoms are constrained by the buttress thread that has a surface contact perpendicular to the barrel reference axis. Figure 12 shows how the degrees of freedoms are constrained by the spherical interface and the buttress thread. Figure 13 shows a zoomed view of the interfaces between the barrels and the thread profile.

\section{Injection molded plastic lens}

The use of injection molding techniques to manufacture plastic lenses offers further possibilities for mounting and alignment when compared to lens made of glass fabricated using traditional grinding and polishing techniques. To provide compact mounting, such as it is required for smartphone 


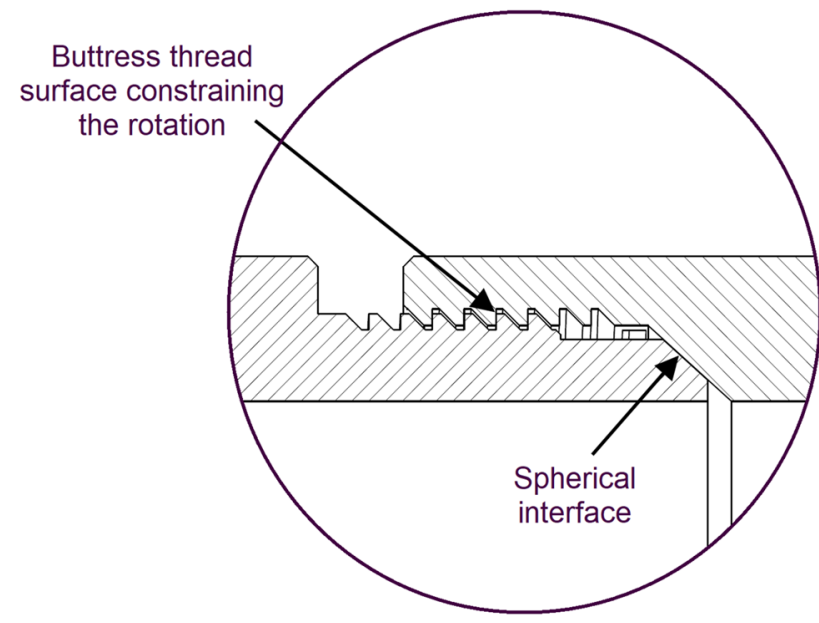

Fig. 13 Barrel stack auto-centering zoomed view

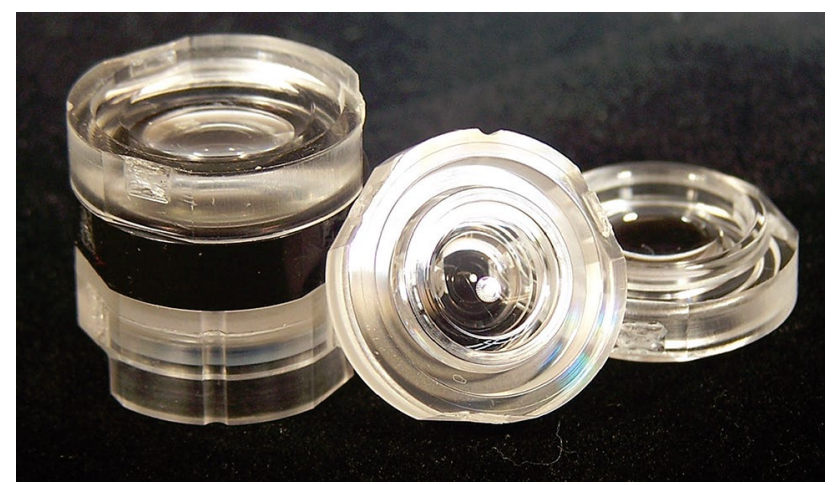

Fig. 14 Integral tapered flange mounting [8]

lens assembly, integral mounting flanges molded with the lens, as shown in Fig. 14, are widely used [8].

The flanges are used to stack the lens on each other to control their axial position. In addition, tapered flanges that engage each other are typically used as centering features, as shown in Fig. 15.

In such case, a tapered protrusion on the first lens is inserted in a tapered cavity on the second lens to provide the centering. This requires to have a minimal clearance between the two mounting interfaces, so that the lenses can be assembled without mechanical interference, which would result in an axial positioning error as well as a rocking movement of the lenses. Moreover, the minimal assembly clearance to avoid mechanical interference is increased by the diameters manufacturing tolerances.

To improve the commonly used methods base on assembly clearance, an innovative concept using toroidal interfaces has been developed. This patent pending method [9] allows a reduction by at least a factor of two the lens centering error compared to classical methods based on radial clearance

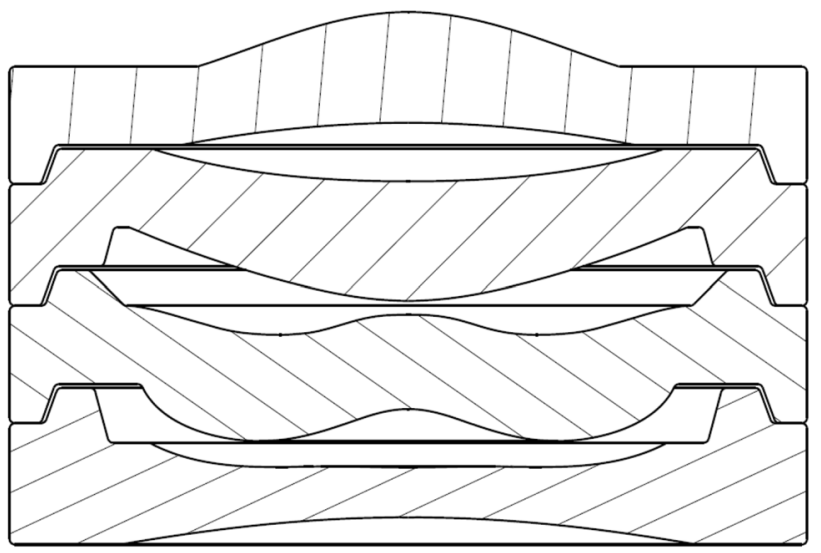

Fig. 15 Lenses centered using tapered flange

fit for the same manufacturing tolerances. This is achieved by the use of a toroidal protrusion that interfaces with a V-groove, as depicted in Fig. 16.

Figure 17 shows how the centering error of lenses is affected by the manufacturing tolerance for assembly methods based on radial clearance fit. For this method, the maximum centering error is the half value of the sum of the minimum diametrical clearance, the outer diameter tolerance, and the inner diameter tolerance.

Figure 18 shows the toroidal diameters manufacturing tolerance interval. Since there is no assembly clearance required to mount the lens properly to each other, the nominal diameter for both toroidal protrusion and toroidal groove is the same and the diameter tolerance is assigned symmetrically from either side of the nominal value. As a result, the maximum centering error is the half value of the diametrical tolerance of the toroidal interfaces.

Figure 19 shows the effect of a variation of diameters between the toroidal protrusion and the groove. The lens is centered on the figure, but not fully constrained because of the clearance in the inner side of the groove.

To be fully constrained, the lens will tend to decenter until a third point of contact is reached on the inner side of the groove, as shown in the left side of Fig. 20. Once fully constrained, the lens is decentered by about half of the difference between the toroidal protrusion and groove diameters.

For the toroidal interface mounting, the protrusion and the groove can both have a diametrical error with respect to the nominal value, but still provide a perfect entering. In fact, as long as the two diameters are the same, the centering will remain conserved. Thus, there are several diameters of the toroidal interfaces that would theoretically result in a perfect centering. This contrast with classical lens centering based on clearance fit, where the minimum centering error will occur only when the inner diameter is at is maximum value and the outer diameter is at is minimum value. As a 
Fig. 16 Toroidal interface for lens centering
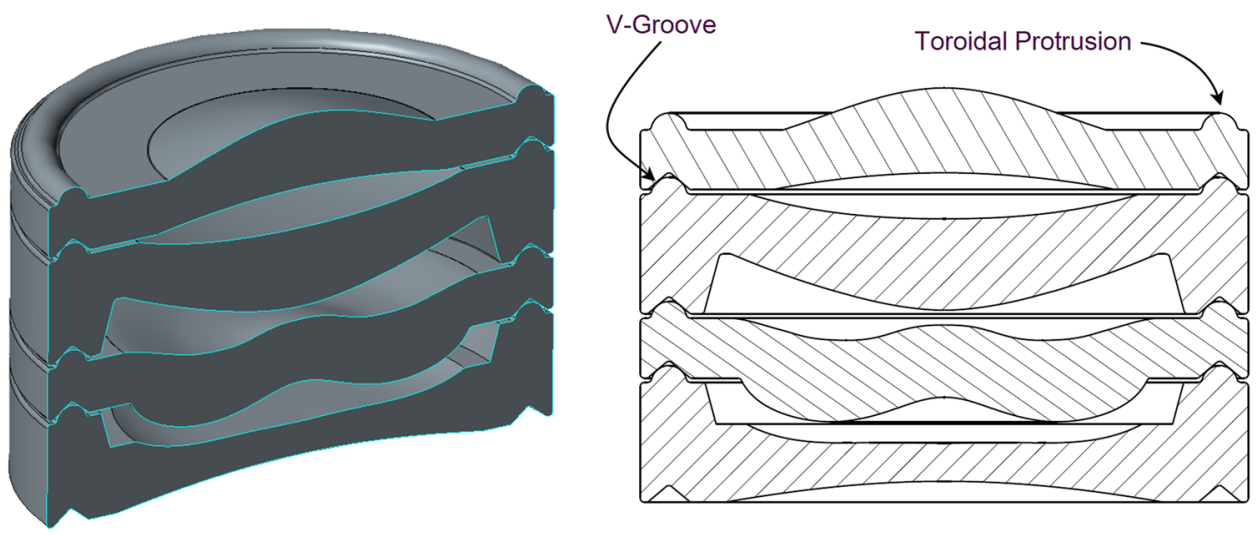

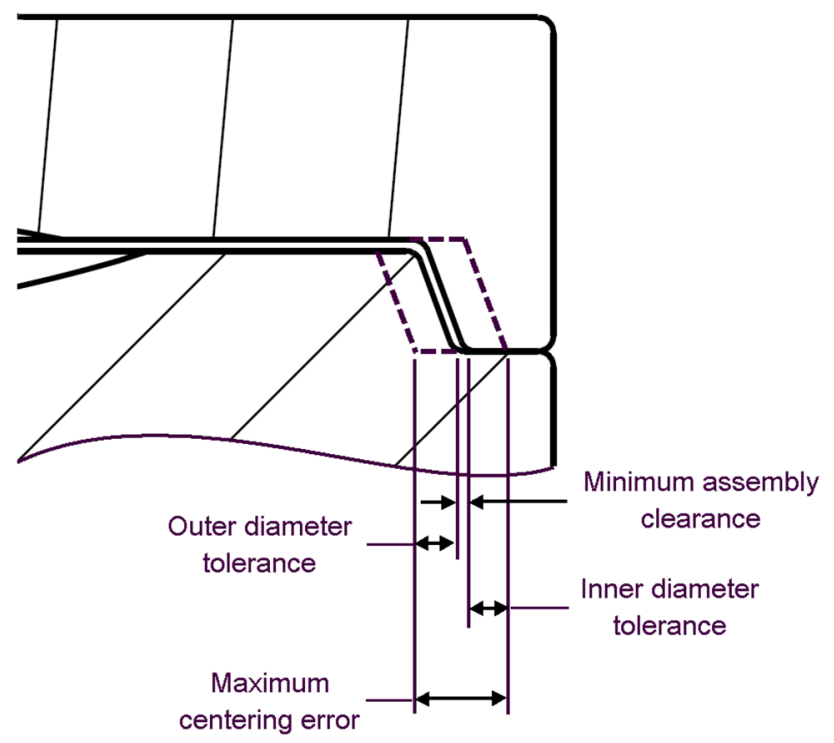

Fig. 17 Centering error based on assembly clearance

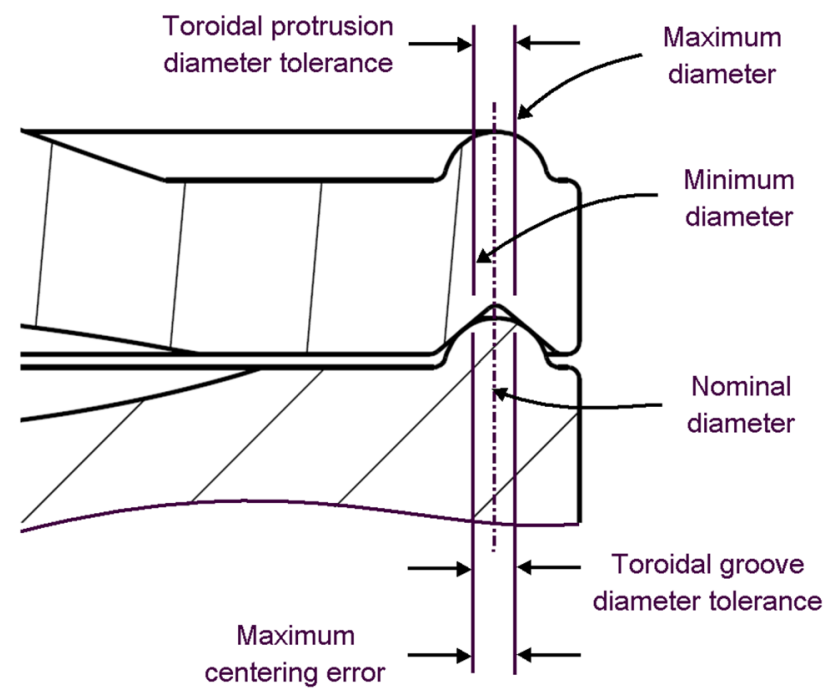

Fig. 18 Centering error using toroidal mounting

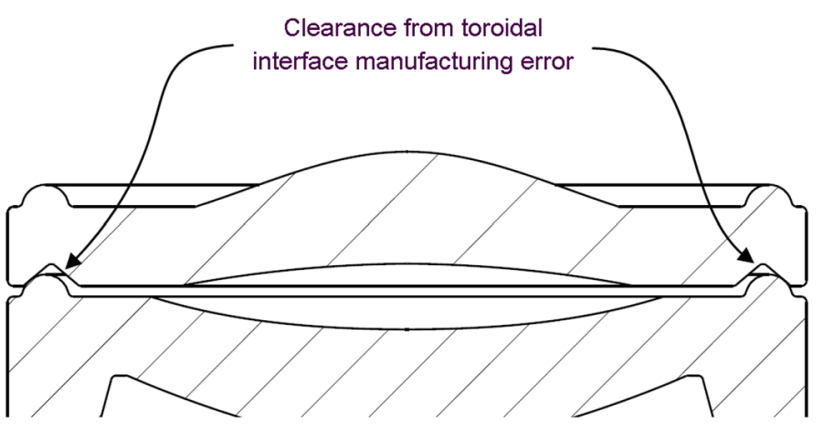

Fig. 19 Toroidal interface with diameters manufacturing errors

result, the maximum centering error for the toroidal mounting method is reduced by a factor of two compared with classical methods based on radial clearance fit, but the statistical centering error improvement is even better.

In precision lens assembly, tilt and distance between the lenses also need to be controlled accurately. Since the toroidal protrusion and the $\mathrm{V}$-groove constrain five degrees of freedom of the lens, manufacturing errors on these surfaces will also affect the tilt and the axial spacing between two lenses. It can be seen from Figs. 19 and 20 that a mismatch of the radii of the toroidal protrusion and of the toroidal groove results rocking movement of the lens. Therefore, there is a tilt imparted to the lens in addition to the centering error described previously. This tilt error can be minimized by the use of a larger V-groove angle. In addition, this tilt error remains moderate and is of the same order as the centering error, unlike traditional centering methods, where a small tilt is typically associated with a larger decentering.

Manufacturing errors on the toroidal section diameter as well as on the V-groove section profile will mostly affect the axial spacing between two lenses. To minimize the axial distance variation between two lenses, a good control of these tolerances is required. Since these flange features are machined into the metal of the mold cavity sets, these manufacturing tolerances are fairly precise. 
Fig. 20 Lens decentered by the toroidal interfaces' diameter manufacturing errors

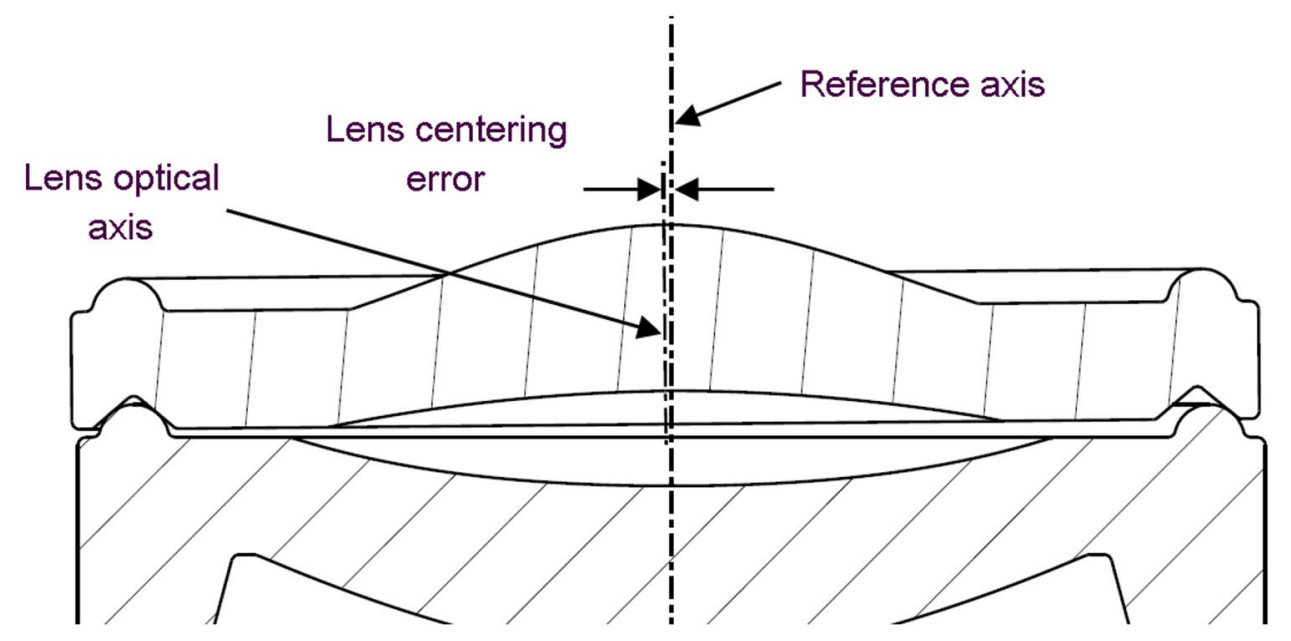

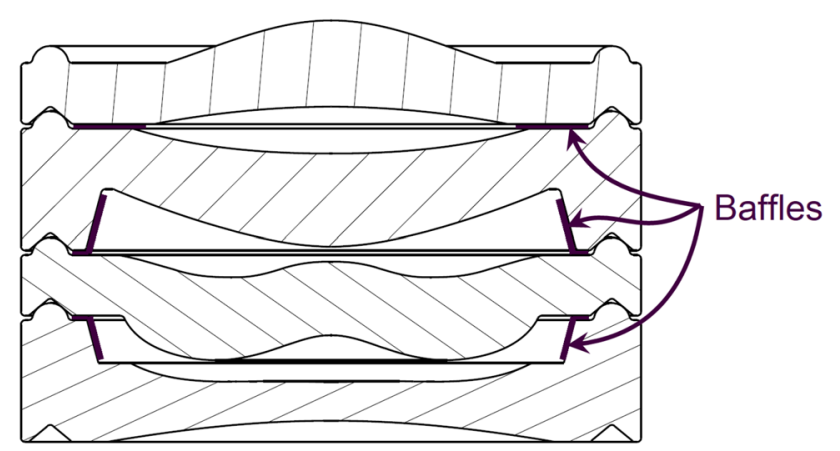

Fig. 21 Thin ring baffles to minimize light reflection

In addition to these centering, tilt, and axial errors, other contributors on the lens positioning are involved as for any other type of mounting interface. Among these errors, there are manufacturing variations associated with the manufacturing processes such as die machining and molding. For example, centering error between the two optical surfaces of a single lens, lens thickness error, and molding distortions are intrinsic to the manufacturing process and are not compensated by the mounting interfaces.

Finally, the integration of mechanical features in plastic optics, while very useful for the mounting, can increase the possibility of creating stray light paths in the optical system. Blocking of stray light paths in plastic lens assembly is usually accomplished by the addition of opaque surfaces [8]. Baffles such as thin annular rings are commonly used in plastic lens assembly to minimize the light reflection. The toroidal interface mounting method allows the use of this baffling method, as shown in Fig. 21. Such aperture rings can be stamped from black Mylar or other thin materials and captured in the gap between the lenses.

\section{Conclusions}

A comprehensive study performed on interaction between lens and mount has resulted in the development of new solutions to mount lenses accurately. This paper summarizes the solutions developed to mount glass and injection molded plastic lenses to improve the centering accuracy of classical methods without the use of active alignment and tight manufacturing tolerances.

Open Access This article is distributed under the terms of the Creative Commons Attribution 4.0 International License (http://creativeco mmons.org/licenses/by/4.0/), which permits unrestricted use, distribution, and reproduction in any medium, provided you give appropriate credit to the original author(s) and the source, provide a link to the Creative Commons license, and indicate if changes were made.

\section{References}

1. Yoder Jr., P.R., Vukobratovich, D.: Opto-Mechanical Systems Design, 4th edn. CRC Press, Boca Raton (2015)

2. Ahmad, A., Lamontagne, F.: Chapter 7: optomechanical tolerancing and error budgets. In: Ahmad, A. (ed.) Handbook of Optomechanical Engineering, 2nd edn. CRC Press, Boca Raton (2017)

3. Trabert, M., Fuchs, U., Kiontke, S.R.: Asphere wedge decenter: what you see is not always what you get. Proc. SPIE 9951, 99510I (2016)

4. Lamontagne, F., Desnoyers, N.: Auto-centering of an optical element within a barrel, US Patent 9,244,245, 26 Jan 2016

5. Lamontagne, F., Desnoyers, N., et al.: Lens auto-centering. Proc. SPIE 9626, 962619 (2015)

6. Lamontagne, F., Fuchs, U., Trabert, M., Möhl, A.: Aspheric lens mounting. Opt. Eng. 57(10), 101708 (2018)

7. Lamontagne, F., Desnoyers, N., Bergeron, G., Cantin, M.: High precision optomechanical assembly using threads as mechanical reference. SPIE 9951, 995107 (2016)

8. Ahmad, A., Schaub, M.: Chapter 5: plastic optics. In: Ahmad, A. (ed.) Handbook of Optomechanical Engineering, 2nd edn. CRC Press, Boca Raton (2017) 
9. Lamontagne, F.: Optical elements with toroidal engagement interfaces and method for assembling such elements, US Patent Application serial no. 2019/0011609 (2019)
Publisher's Note Springer Nature remains neutral with regard to jurisdictional claims in published maps and institutional affiliations. 